\title{
SABERES EDUCATIVOS MAPUCHES: APORTES EPISTÉMICOS PARA UN ENFOQUE DE EDUCACIÓN INTERCULTURAL ${ }^{*}$
}

\author{
MAPUCHE EDUCATION KNOWLEDGE: EPISTEMIC CONTRIBUTIONS \\ FOR AN INTERCULTURAL EDUCATIONAL APPROACH
}

\author{
Daniel Quilaqueo ${ }^{1}$, Segundo Quintriqueo ${ }^{1}$, Héctor Torres ${ }^{2}$ y Gerardo Muñoz ${ }^{1}$
}

\begin{abstract}
Este artículo expone saberes educativos mapuches que forman parte de la educación familiar. El objetivo es contribuir con el debate epistémico de contenidos educativos indígenas de educación intercultural, para superar su desconocimiento en escuelas situadas en los contextos de vida mapuche de hoy día. Se observa que el desconocimiento de los saberes educativos mapuches dificulta la implementación de un enfoque educativo más adaptado a los conocimientos socioculturales, para los alumnos de origen tanto mapuche como no mapuche. En efecto, la escolarización de niños mapuches y no mapuches se ha basado casi exclusivamente en un currículum monocultural de la sociedad occidental. La metodología se fundamenta en la investigación educativa, desde una perspectiva cualitativa mediante una muestra de 16 kimches (sabios). Los resultados revelan categorías de conocimiento vinculadas a conceptos de tiempo y espacio del ciclo anual, conceptos de procedencia geográfica-territorial (tuwün) y ascendencia parental (küpan). Cada uno de estos aspectos es incorporado por la familia mapuche en el proceso de formación de los niños y jóvenes. Se concluye que estos conocimientos pueden constituirse en un aporte epistémico que favorezca una educación contextualizada y potencie la formación de los alumnos a partir del diálogo de saberes desde un enfoque intercultural.
\end{abstract}

Palabras claves: saberes educativos mapuches, educación intercultural, diálogo de saberes.

This article presents Mapuche education knowledge that forms part of a family's education. The aim is to contribute to the epistemic debate on the indigenous educational content of intercultural education for the purpose of overcoming ignorance of this content in schools located within contemporary Mapuche life. Ignorance of the Mapuche's educational knowledge hinders the implementation of an educational approach best suited to the sociocultural knowledge, for both Mapuche and non-Mapuche students. In fact, the schooling of both Mapuche and non-Mapuche children has been based almost exclusively on the monocultural curriculum of Western society. The methodology is based on educational research from a qualitative perspective through interviews with a sample of 16 kimches (sages). The results reveal categories of knowledge linked to concepts of time and space in the annual cycle, concepts of geographical-territorial origin (tuwün), and parental descent (küpan). The Mapuche family incorporates each of these aspects into the education of children and adolescents. It is concluded that this knowledge may constitute an epistemic contribution that favors a contextualized education and strengthens the education of students through the dialogue of knowledge systems from an intercultural approach.

Key words: Mapuche knowledge, intercultural education, dialogue of knowledge systems.

En este artículo se exponen algunas categorías de contenidos conceptuales y procedimentales que componen los saberes educativos mapuches, desde la perspectiva de los kimches (sabios) de la región de La Araucanía, Chile. Estos saberes constituyen un aporte epistémico para fundamentar un enfoque educativo intercultural ante la monoculturalidad escolar (Quintriqueo 2010). El problema abordado se refiere, por una parte, al quiebre de la identidad sociocultural de la población mapuche y a la pérdida progresiva del saber cultural y educativo tradicional de las nuevas generaciones y, por otra, al desconocimiento social y cultural que ha generado la escuela entre los alumnos de origen no mapuche. En consecuencia, los alumnos de origen mapuche deben asumir la coexistencia de dos modelos educativos: el familiar mapuche y el escolar.

La educación familiar se reconoce como la acción educativa donde los niños y los jóvenes se disponen para el aprendizaje de saberes y

\footnotetext{
1 Universidad Católica de Temuco, Chile, Manuel Montt № 56, Temuco. Casilla 15-D, Temuco, Chile. dquilaq@uct.cl; squintri@uct.cl; geramaster@gmail.com

2 Centre Interuniversitaire d'Études et de Recherches Autochtones (CIÉRA), Université Laval, Québec, Canada. hector.torres-cuevas.1@ulaval.ca
} 
conocimientos, como reflejo de las construcciones sociales y cognitivas del grupo familiar (Beillerot y Mosconi 2006; Quilaqueo et al. 2005). Por su parte, la educación escolar se reconoce como medio de enseñanza del conocimiento occidental, con un carácter monocultural y centralizado, cuya finalidad ha sido la inclusión de los mapuches a la sociedad nacional (Quilaqueo y Quintriqueo 2010; Quintriqueo y McGinity 2009).

El desconocimiento y exclusión de los saberes educativos mapuches en la escuela, por parte de las instituciones estatales, sería el resultado de los tipos de relaciones que se han establecido históricamente entre los mapuches y los no mapuches (Quilaqueo 2009). La educación escolar, desde entonces, se ha fundamentado en un currículum escolar descontextualizado que ha favorecido la reproducción de modelos homogenizadores para mantener las relaciones de poder y dominación que tienden a reducir la diversidad sociocultural de las comunidades indígenas al llamado mundo cultural mestizo (Fornet-Betancourt 2004). De este modo, los procesos escolares continúan cerrados a la posibilidad de transformarse de manera contextualizada e intercultural. Esto conduce, por ejemplo, a una formación que provoca el desarraigo sociocultural y la desvalorización del conocimiento educativo mapuche entre niños y jóvenes. Desde este argumento, los padres, abuelos y kimches son los principales testigos del debilitamiento de la enseñanza del conocimiento sociocultural entre las familias mapuches.

En este trabajo, para ejemplificar algunas categorías de saberes educativos mapuches presentes en la educación familiar, se exponen los conceptos de tiempo y espacio del ciclo anual, los conceptos de procedencia geográfico-territorial (tuwün) y la ascendencia parental (küpan) que se enseñan a niños y jóvenes como contenidos actitudinales y procedimentales (Quilaqueo et al. 2011). La categorización que se establece tiene como objetivo acercar a la comprensión de cómo se forma una persona desde la racionalidad educativa mapuche (Quilaqueo 2012). De esta forma se busca aportar conocimientos socioculturales que puedan ser incorporados en un currículum escolar con enfoque educativo intercultural. Su no incorporación al currículum escolar, en consecuencia, obstaculiza el desarrollo de la educación sustentada en el diálogo de saberes ${ }^{1}$. Para la familia mapuche, los saberes educativos se sustentan en conocimientos sociales y culturales representados en la memoria social de los grupos familiares y comunidades territoriales. En este sentido se presentan conocimientos construidos durante la formación de persona mapuche como aportes cognoscitivos para superar el desconocimiento epistemológico que hay en la escuela acerca de los contenidos sociales, culturales y de la naturaleza enseñados por la familia mapuche.

\section{Marco de Referencia}

En escuelas tanto rurales como urbanas de contextos mapuches, la formación de niños y jóvenes se ha caracterizado por estar fundamentada en un currículum escolar monocultural que ha negado el saber educativo mapuche (Quilaqueo 2006; Quilaqueo y Merino 2003; Quintriqueo y McGinity 2009). Aunque en estos estudios los autores no hacen un análisis histórico de la escuela chilena, revelan tres principios que han sido la base de la escolarización y de la inclusión de los mapuches a la sociedad nacional. Estos son: combatir la barbarie del mapuche, civilizarlo y convertirlo en ciudadano. Según Donoso (2008), estos principios se relacionan con la intención predominante de eliminar las prácticas socioculturales ancestrales mapuches, principalmente durante las cuatro primeras décadas del siglo XIX, pero que perdura hasta hoy día. Así, en el proceso de escolarización, las prácticas culturales mapuches se consideraban como conocimientos que entorpecían la incorporación del mapuche a la sociedad chilena. A partir de esto, se puede deducir que la base de la formación escolar focalizó su acción en la conversión del mapuche a un ciudadano civilizado, cristiano y chileno, según los parámetros de la racionalidad occidental (Flores y Azócar 2006). Lo anterior se consolidó durante el siglo XX mediante la escuela, que en términos de Giroux (2004:214), “eran generalmente campos de entrenamiento para el desarrollo del carácter y para el control económico y social".

De acuerdo con las características de las escuelas establecidas en contexto mapuche, la formación de niños y jóvenes se ha llevado a cabo en una escuela que entrega saberes muy distantes de los que se enseñan en el medio familiar mapuche. Esta distancia entre la formación que entrega la familia y la escuela se fundamenta en una visión epistemológica sobre el conocimiento y las finalidades educativas (Quintriqueo y Torres 2013). Así, la escuela se ha caracterizado por validar contenidos y finalidades 
educativas que permitirían a los alumnos lograr supuestos éxitos laborales y económicos fuera de su contexto de vida, donde los conocimientos propios quedan ocultos, por su desprecio epistemológico (Gasché 2010; Tubino 2011). En esa perspectiva, la lógica que utiliza la escuela al incorporar valores y conocimientos que entran en conflicto con la enseñanza familiar de niños y jóvenes mapuches ha favorecido el desarraigo sociocultural y la desvalorización del modo de vida mapuche (Gasché 2010a; Quilaqueo y Quintriqueo 2010). En este sentido, la escuela ha funcionado bajo el modelo de colonialidad del saber. Es decir, que ha fundado su acción educativa en marcos de referencias que no se despojan de las marcas legadas de la herencia colonial, enseñándose saberes centrados epistémicamente en conceptos, procedimientos y valores eurocéntricos. Walsh (2008) señala que la colonialidad del saber es particularmente evidente en el sistema educativo (desde la escuela hasta la universidad) donde se eleva el conocimiento y la ciencia europea como el marco científico-académicointelectual. De esta manera, se ha favorecido la hegemonía del conocimiento de raíz eurocéntrica en desmedro de los conocimientos construidos por las comunidades indígenas, reproduciéndose los modos de pensamiento coloniales (Maldonado 2007). Al respecto, se requiere de un análisis crítico, ya que la formación entregada por los docentes en la escuela no se sustenta en el diálogo de saberes, es decir, en la generación de una articulación de distintas lógicas de conocimiento, que discutan las posiciones universales y hegemónicas, avanzando en la incorporación de saberes educativos mapuches como alternativa al modelo monocultural que imponen los contenidos del currículum escolar. En efecto, la educación escolar entrega una formación que, por un lado, no potencia el fortalecimiento de la identidad en los estudiantes de origen mapuche y, por otro, no permite la enseñanza del conocimiento sociocultural y educativo mapuche a los estudiantes no mapuches. De acuerdo con lo expresado por Freire (1997:130), es clave que los docentes puedan "vivir la apertura respetuosa a los otros y, de vez en cuando, de acuerdo con el momento, tomar la propia práctica de apertura al otro como objeto de reflexión crítica". Por lo tanto, los profesores en contextos de relaciones interculturales tienen un gran desafío, primero, la necesidad de cuestionar la hegemonía del conocimiento occidental; segundo, proyectar un trabajo educativo articulado con los padres de familia y el conjunto de la comunidad; tercero, llevar a la práctica procesos educativos que asuman el compromiso de formar ciudadanos interculturales. Es decir, personas que se dispongan a superar el racismo, los estereotipos y discriminación presentes en la sociedad, capaz de interactuar en base al conocimiento de distintos saberes (indígena, campesino, occidental y científico), abierto a discutir los marcos epistémicos hegemónicos y romper con las estructuras rígidas, mecánicas y lineales de la escuela.

Surge la necesidad de configurar una educación definida a partir de los marcos de referencias que son la base de la educación familiar mapuche, para así superar la naturaleza descontextualizada del aprendizaje escolar que oculta el valor pedagógico del conocimiento educativo propio de sus estudiantes (Quilaqueo et al. 2011; Quintriqueo y Cárdenas 2010). De esta manera, se plantea como desafío abrir la formación a un diálogo en contextos interculturales, a partir de una "educación liberadora y humanizante, que rompa con las condiciones de dominación impuestas por el intelectualismo alienante" (Freire 2007:131). Para ello, es necesario cuestionar las condiciones que han definido la institucionalización de la propuesta de educación intercultural bilingüe por parte del Estado, porque este ha limitado la capacidad de acción y transformación de los procesos educativos al incorporar conocimientos indígenas y locales que funcionan con la lógica de un currículum escolar monocultural (Forno et al. 2009). En definitiva, se asume que la educación intercultural debe ser comprendida como un proceso transformador de la práctica educativa, que pone en discusión los modelos, racionalidades, conocimientos y concepciones de persona que han definido la enseñanza tradicional de la escuela (Quilaqueo et al. 2011).

Particularmente en el contexto mapuche, se comprende que la implementación de la educación intercultural necesita que los actores que participan del proceso educativo (estudiantes-docentes-familias) se despojen de la condición dominante-dominado. Bajo este marco, resulta relevante discutir las asimetrías valorativas provocadas por el racismo y la discriminación (Schmelkes 2009), que favorecen la negación y ocultamiento de los conocimientos que forman parte de los contextos de vida de niños y jóvenes mapuches (Quintriqueo 2010). Para ello, una propuesta pedagógica intercultural debiera asentarse en los siguientes aspectos: (1) el interaprendizaje 
entre los actores participantes del proceso educativo. Para esto, debe existir un acercamiento entre los sujetos, que permita disponer de espacios y tiempos de trabajo, para que profesores y padres de familia no generen nuevas barreras o caigan en prácticas funcionales impregnadas de la mecánica de la cultura escolar. Es posible ajustar la escuela a los tiempos del contexto local, girar epistémicamente en la dirección de una educación articuladora y no segregadora, donde los proyectos educativos institucionales no son solamente norma, sino que posibilidad de acción y construcción colaborativa del conocimiento en la relación escuela-familia y comunidad; (2) Superar el desconocimiento del Otro, que los docentes puedan conocer la historia de la comunidad, los hitos y lugares significativos en la vida cotidiana de los alumnos, padres, abuelos $\mathrm{y}$, a su vez, los miembros de la comunidad se dispongan a compartir sus conocimientos, a expresar sus sentimientos, construir un sentido de la escuela para la vida mapuche actual y que puedan ser valorados en la formación de los niños y jóvenes; (3) Confrontar la estructura de un modelo de educación intercultural, limitado a aspectos funcionales del saber indígena. Por ejemplo, una escuela no es intercultural por incluir en su programación curricular la celebración del we xipantu (año nuevo mapuche). Al contrario, esto puede terminar por generar la pérdida del sentido, valor y simbolismo que engloba en el mundo de vida mapuche, el cambio de ciclo anual, transformándose en una fecha más del calendario escolar; un día dentro de la agenda del tiempo occidental. Por eso, un programa de educación intercultural cuando supera los marcos funcionales, se acerca a una educación que puede configurarse desde distintas dimensiones epistémicas, transgresora de la mecánica que tiende a folklorizar el saber del Otro; (4) La formación intercultural no puede seguir anclada principalmente al contexto de las comunidades indígenas, a los sectores rurales o los sectores urbanos, donde hay mayor población indígena asociada a pobreza y subdesarrollo. No es posible avanzar en cambios sociales, culturales y educativos, que permitan al conjunto de la población no indígena en superar su terror por las diferencias, si continúan siendo excluidos de procesos de formación intercultural. Continuar la focalización de la educación intercultural en el mundo indígena es mantener la reproducción de políticas que no son capaces de romper el modelo monocultural de base eurocéntrica que opera en las escuelas en Chile, lo cual implica seguir implícitamente diciendo que el problema son los indígenas, por no lograr integrarse a la gran familia chilena; y (5) Se requiere el reconocimiento de una doble o múltiple comprensión del concepto de persona, naturaleza, sociedad y espiritualidad. Mantener una comprensión unidimensional de tipo occidental de lo que significa ser persona, de la relación que se establece con la naturaleza o del significado que tienen las relaciones sociales y del sentido de la espiritualidad solo alimenta el desplazamiento a segundo plano de los marcos epistémicos de los saberes propios, tanto indígenas como campesinos (Gasché 2002; Tubino 2011).

En consecuencia, se reconoce que la educación escolar necesita abrirse a una relación dialógica que permita construir finalidades y contenidos educativos que articulen los conocimientos disciplinares y los conocimientos mapuches (Quintriqueo 2010). Sin embargo, para lograr lo señalado se requiere la descolonización de las ciencias, del saber y del ser (Walsh 2005). Es decir, se necesita transformar las estructuras de poder que han perpetuado sistemas de conocimiento hegemónicos que han sido reproducidos por las instituciones escolares. De esta forma se propiciaría lo que se define como una articulación intercultural de contenidos, que busca superar la desigualdad de oportunidades expresada en una formación escolar monocultural (Gasché 2010a; Quilaqueo 2010). Esto se concibe como una condición vital, tanto en el caso mapuche como en el de otros pueblos indígenas de América Latina, porque para superar el nivel técnico-discursivo de las políticas educativas oficiales que han mantenido las relaciones de desigualdad y la resistencia del medio escolar, es necesario implementar una educación intercultural que incorpore contenidos socioculturales propios de los pueblos indígenas a los procesos de enseñanza escolar (Bertely 2006; Quilaqueo y Quintriqueo 2008; Quilaqueo et al. 2011).

Desde este enfoque, el diálogo de saberes se convierte en una alternativa que favorecería los procesos educativos de manera más contextualizada. Según Pérez y Alfonzo (2008), esto constituye una forma de interacción intencionada entre los docentes, los alumnos y la comunidad, con el fin de expresar el saber propio frente al de los demás, independiente de su carácter de popular, local o científico. En este sentido, se transforma en una red de aportes cognitivos que no busca la marginación 
de los saberes y conocimientos construidos con lógicas particulares de conocimientos. Es decir, se fundamenta en la diversidad de posiciones y perspectivas existentes sobre un tema en común por medio de relaciones intersubjetivas. De esta forma, dada la naturaleza de las condiciones preliminares para la existencia de un diálogo de saberes, es preciso que existan docentes que realicen prácticas pedagógicas abiertas y reflexivas, fundamentadas en la construcción colaborativa del conocimiento (Da Silveira et al. 2002; Quilaqueo 2007).

El diálogo de saberes se fundamenta en la valoración de los patrimonios culturales de la sociedad chilena no indígena e indígena. Sin embargo, el mundo occidental ha validado en exclusividad el conocimiento científico y justifica la marginación de cualquier otro saber construido desde lógicas distintas (Quilaqueo 2006). Lo anterior se fundamenta en las representaciones sociales como razonamiento que busca comprender una realidad y su posterior comunicación (Moscovici 1983). De esta manera, es factible comprender que el conocimiento mapuche, como representación social y racionalización propia de fenómenos específicos, tiene la validez e importancia para su transmisión en contextos escolares (Quilaqueo 2012). Entonces, en el marco de la educación escolar intercultural, el diálogo de saberes se refiere al tratamiento analítico de los contenidos educativos mapuches y curriculares, sin marginarlos de su marco epistemológico para el currículum escolar, valorando y validando a ambos por igual.

\section{Metodología}

La metodología utilizada se fundamenta en la Investigación Educativa, desde una perspectiva cualitativa, y se orienta hacia la búsqueda de conocimientos científicos mediante un conjunto de métodos rigurosos y sistemáticos que permitan comprender los fenómenos educativos (Wallen y Fraenkel 2001; Bisquerra 2004; McMillan y Schumacher 2005). De esta forma, se indagó en la lógica mapuche con el fin de aportar conocimientos para la construcción de una educación intercultural sustentada en el diálogo de saberes, que responda a las características socioculturales de niños y jóvenes mapuches y no mapuches. Con este propósito, se desarrolló un estudio de casos de tipo colectivo (Stake 2005) y se seleccionó a los kimches (sabios) en tanto casos singulares porque poseen saberes educativos mapuches, lo que fue un aporte para la descripción de los contenidos como de la forma en que se han construido.

La investigación se realizó en localidades rurales $^{2}$ de la región de La Araucanía que se ubica geográficamente en la zona Sur de Chile. Al respecto, para el trabajo de campo fueron consideradas comunidades ubicadas en tres áreas territoriales: Wenteche; Nagche y Pewenche. De este modo, las comunidades de estas territorialidades seleccionadas permitieron ingresar al mundo de la vida cotidiana en el que se desarrolla la formación de los niños y jóvenes mapuches. Se utilizó una muestra intencional, no probabilística, de 16 kimches, que se seleccionaron como casos típicos según los criterios de origen étnico, género y ubicación geográfica. $\mathrm{Al}$ respecto, el origen étnico indica que los kimches son personas reconocidas por su origen paterno denominado küpan y materno denominado tuwün. De igual forma, se escogieron kimches hombres y mujeres con el objeto de analizar sus discursos respecto de los saberes educativos mapuches. Por último, la ubicación geográfica se refiere al modo cómo los kimches contextualizan el conocimiento, ya que históricamente han sido reconocidos según el tipo de territorialidad desde una concepción de colectividad histórica (Quilaqueo 2007). Para acceder a los participantes de este estudio, los kimches, considerando los criterios mencionados, se trabajó con un facilitador o informante clave que era miembro de cada comunidad y que trabajaba en la escuela.

Para la recolección de datos se utilizó como instrumento la entrevista semiestructurada, que se aplicó en castellano y en lengua mapuche (mapunzugun). Las entrevistas estaban constituidas por un guion en torno al tema, que permitió obtener información de los conocimientos enseñados a los niños y jóvenes (Bisquerra 2000; Corbetta 2003), y tuvieron una duración de 45 a 60 minutos. Asimismo, fueron registradas en formato de audio digital, previa revisión y firma de un consentimiento informado de los kimches $\mathrm{y}$, posteriormente, transcritas para realizar los análisis. Con el propósito de identificar y diferenciar la entrevista de cada kimche, se utilizó como nomenclatura el símbolo $\mathrm{K}$ (Kimche), el número de la entrevista y la sigla correspondiente al lugar de pertenencia del entrevistado (Ejemplo: L=Lipun, Q=Quilquen, $\mathrm{M}=$ Maquehue, $\mathrm{Ra}=$ Ragintulewfu, $\mathrm{C}=$ Cayaqui, $\mathrm{Ch}=$ Chumil, $\mathrm{Ll}=$ Llamuco y $\mathrm{Re}=$ Repocura). 
El procesamiento de los datos se realizó utilizando la propuesta del análisis de contenido (Ruiz Olabuénaga 1996; Quivy y Campenhoudt 1998). Así, el análisis siguió un proceso riguroso de lectura de los testimonios de los kimches, lo que permitió la identificación de unidades de registro o códigos que fueron sistematizados y clasificados en categorías de contenido. De esta forma, la codificación se inició considerando un sistema abierto de categorías, que fueron comparadas, refinadas y saturadas con el propósito de llegar a la explicitación de los conocimientos educativos mapuches. Dichos conocimientos pueden contribuir a salir de la hegemonía epistemológica occidental, al aportar con otra perspectiva respecto de la valoración de las relaciones familiares, a la construcción del conocimiento del tiempo, a la relación con la naturaleza, a la importancia de estar atento no solo de los aspectos concretos de la vida, sino que también de los aspectos espirituales. Por último, para agilizar el tratamiento de los datos, se utilizó el software de análisis cualitativo Atlas.ti 5.0.

\section{Resultados}

Los resultados se organizaron en las siguientes categorías de contenidos educativos: contenidos del küpan, contenidos del tuwün y contenidos de la organización del ciclo anual.

\section{Contenidos educativos del küpan}

El concepto küpan indica la ascendencia paterna de un individuo, vinculado a su lugar de asentamiento y al estatus social que ocupa, lo que se vincula, por ejemplo, con el oficio de los miembros del grupo familiar como elementos que definen las características de la personalidad de un individuo en relación con sus antepasados. En efecto, es un componente esencial de la identidad sociocultural mapuche, que se logra por medio del aprendizaje de los siguientes contenidos educativos.

El primero considera a la familia como raíz de procedencia, ya que cada persona procede de cuatro raíces que lo sustentan en cuanto al conocimiento utilizado, a las prácticas culturales realizadas y a los roles ejercidos en la organización comunitaria. Dicho conocimiento es expresado en mapunzugun como meli folil küpan, es decir, cuatro raíces de ascendencia referidas a los abuelos paternos y maternos. Al respecto, un kimche señala: "Como fui hijo de madre soltera, solo tuve una parte de mis raíces. Estos fueron mis difuntos abuelos Manuel y mi abuelita Lorenza [...]" (K12Re [206:206]). En este discurso se constata que la validez de los abuelos, como raíz parental, se relaciona con su participación en la formación de las nuevas generaciones, por lo que su ausencia se entiende como limitación de la formación de los niños y jóvenes para acceder al conocimiento educativo mapuche.

El segundo contenido se refiere a la denominación de los distintos grados de parentesco de los integrantes del grupo familiar, cuya importancia está en que permite el conocimiento de los miembros de la familia extendida. De esta manera, se propicia el desarrollo de interacciones interpersonales fundamentadas en el respeto y descendencia con el vínculo parental. Un kimche expresa que los conceptos de "maje, palu, fücha, chaw y ñuke" (K13Re [60:60]), se refieren al tío, tía, papá y mamá respectivamente. Esto implica conocer el grado de parentesco de los integrantes del grupo familiar, ya que permite establecer uniones matrimoniales con individuos provenientes de otros grupos familiares, lo que se expresa como una norma fundamental para el matrimonio, con el fin de no perder el estatus ante la comunidad.

El tercer contenido se refiere a las representaciones sociales de las familias en la organización comunitaria mapuche, que son valoradas en relación con la cualidad de respeto y conocimientos socioculturales mapuches que poseen. A partir de esto se reconocen buenas y malas influencias, que serían heredadas por los miembros del grupo familiar, hecho que influye directamente en las relaciones que se generan entre las familias. Por tal motivo, pertenecer a una familia bien evaluada social y culturalmente es un apoyo vital, ya que incide en la calidad de la formación de los niños y jóvenes. Esto se observa cuando se expresa: "yo tengo tres hijos, ellos están casados, son respetuosos, no son tímidos, no son pobres y no deben serlo porque tienen buena ascendencia" (K16L1 [82:82]). Al contrario, quienes provienen de familias evaluadas negativamente por los integrantes de una comunidad, encuentran dificultades al establecer relaciones sociales, ya que a los niños y jóvenes se les enseña a alejarse de las personas mal evaluadas por la comunidad. Estos contenidos formativos permiten regular la inserción de las nuevas generaciones en la vida social y en la construcción de la identidad personal y sociocultural. 


\section{Contenidos educativos del tuwïn}

En los discursos sociales actuales de los miembros de las comunidades, el tuwün se refiere principalmente al origen territorial y familiar de un individuo, ya que se relaciona con conocimientos sobre las características de su territorialidad y la interacción de las personas con el medio natural, el medio familiar y cultural. De este concepto se desprenden los siguientes contenidos educativos.

El primer contenido se refiere al lugar de asentamiento familiar que es la acepción más utilizada del tuwün, porque propicia la formación de la identidad y pertenencia de un individuo a su espacio de nacimiento y crianza, lo que revela así la interrelación hombre-naturaleza. Un kimche expresa al respecto que el "tuwün es el lugar de donde viene una persona: el lof [comunidad]. Si yo voy a otro lugar, debo decir de dónde vengo" (K11L1 [54:54]). Este ejemplo ratifica que el tuwün se refiere al origen territorial de una persona y que es expresado en los actos de visita a personas de lugares distintos del propio. Cabe señalar que como producto de la filiación paterna, actualmente utilizada por la familia mapuche, el tuwün se vincula con la localidad de asentamiento del padre.

Un segundo contenido se refiere al parentesco como inatuwün, que significa la indagación, reflexión y prácticas socioculturales para conocer la ascendencia materna y territorial de las personas, como un mecanismo de relación dialógica de los sujetos con el presente, el pasado y el futuro. Los contenidos centrales en la educación familiar es el yamün, que se refiere al respeto entre parientes, el piwkeyewün, entendido como una representación del valor a la relación de parentesco, el logkontuku küpan ka tuwün, como un proceso de representación cognitiva del conocimiento conceptual sobre el parentesco. En este proceso, el mapunzugun se constituye en un mecanismo de expresión, de comunicación de contenidos sobre el parentesco, a través del gübam, a un método educativo para la transmisión situacional del conocimiento, lo cual incluye la memoria y la transformación social en el contexto de la familia y comunidad mapuche.

Un tercer contenido de saberes educativos se refiere al territorio, como representación cultural del tuwün, que permite señalar los espacios específicos al interior de la comunidad, simbolizados en fuerzas espirituales denominadas en lengua mapuche como gen. Por lo general, estos espacios son ríos, vertientes y humedales que deben ser respetados y valorados en relación con la fuerza espiritual gen. Un kimche señala: "por acá tenemos una vertiente donde hay personas que han visto aparecer cosas extrañas... A mí me han enseñado que a la vertiente no se puede ir al mediodía, ya que puede aparecer un culebrón" (K14Ch [78:78]). El testimonio evidencia la enseñanza adquirida por los niños como medio de cuidado, ante el potencial peligro que presenta un río o una vertiente, para que no se transgredan las normas de comportamiento establecidas por la comunidad y que, al mismo tiempo, los niños se desarrollen mejor física y espiritualmente. Aquí, la enseñanza se realiza mediante la leyenda de la aparición de un culebrón. En síntesis, el tuwün propicia principalmente el aprendizaje del lugar de procedencia de una persona, en el que se ha asentado su familia por generaciones y que incluye contenidos educativos como las características geográficas y las representaciones sociales construidas por la familia y comunidad respecto de los espacios para proteger y cuidar tanto a los niños como a la naturaleza.

\section{Contenidos de la organización del ciclo anual}

Respecto de la organización del ciclo anual, se identifica la utilización de conceptos que permiten construir un calendario estacional relacionado con los ciclos de la naturaleza y las actividades sociales y culturales de la familia. De esta forma, los conocimientos se relacionan con el pukem, que es equivalente a parte del otoño y el invierno, el pewü, que es equivalente a la primavera, y el walüg, que es equivalente al verano y a parte del otoño. Entonces, de acuerdo con los testimonios de los kimches, se identifican tres estaciones marcadas por el fin y el comienzo del ciclo anual denominado we xipantu, fecha que coincide con el solsticio de invierno. Estas tres estaciones que se han identificado están caracterizadas por tres dimensiones: social, espiritual y natural.

El pukem se considera como la primera estación del año, es el tiempo que marca el fin y el comienzo de un nuevo ciclo anual y se caracteriza por fenómenos naturales relacionados con el inicio de las lluvias, las bajas temperaturas y la caída de gran cantidad de nieve en los sectores de la cordillera de los Andes. La dimensión social del pukem se refiere a las actividades familiares, principalmente, en torno a los trabajos de la siembra y a la celebración del we xipantu. Lo espiritual se 
vincula con aspectos simbólicos relacionados con la fuerza de la naturaleza, que se manifiestan en la celebración del we xipantu, y lo natural se asocia con el tiempo que provoca el enfriamiento y la limpieza de la naturaleza por efecto de las condiciones climáticas. Un kimche señala: "normalmente debe llegar el mal tiempo: el viento, el trueno y, cuando parece que mejora el tiempo, cae la helada y ahí hay que estar en la casa" (K9C [36:39]). Si bien las condiciones climáticas son difíciles de sobrellevar por su impacto en la vida de la comunidad, estas no son vistas como algo negativo, debido a que se consideran como necesarias para que se mantenga el equilibrio de la naturaleza.

El pewü se define como la estación del brote de la vegetación y se reconoce como el tiempo del renacer de la naturaleza, lo que se evidencia en el brote de los frutos de los vegetales herbáceos, árboles y arbustos. Al respecto, una kimche señala: "en la primavera brotan los árboles, se vuelven verdes los pastizales, toda la vegetación vuelve a crecer, en los bosques y en el valle lelfün" (K2L [133:133]). Desde la perspectiva climática, el pewü se define como un período de cambio del tiempo, que se caracteriza por la disminución del frío y de las lluvias y por la llegada de días más soleados y calurosos. Este período es muy esperado por los miembros de la comunidad, porque anuncia el tiempo de la cosecha de los productos sembrados. Asimismo, se caracteriza por observarse un aumento en la temperatura y una progresiva extensión del día y, por ende, su claridad, lo que favorece el crecimiento de la vegetación y las actividades sociales de los jóvenes.

El walüg se refiere al tiempo de cosecha, que equivale a la estación de verano del calendario occidental y se considera como el período de abundancia de alimentos en el que se registran los días más calurosos y largos del año. Al respecto, un kimche señala: "En el walüg se cosecha, ahí encontramos todo tipo de alimentos, hay mucha fruta, uno no lo pasa mal, ya que no se pasa hambre" (K1L [191:191]). El testimonio permite comprender que el walüg marca la etapa en la que se obtienen los alimentos que han sido sembrados y los que se recolectan, como el piñón en las comunidades pehuenches. De esta forma, este período se reconoce como un tiempo que permite la interacción entre los miembros de la comunidad en la realización de actividades como la cosecha. Por otra parte, es un período de agradecimiento de las personas a las fuerzas espirituales y sobrenaturales que simbolizan, de acuerdo con sus creencias, la posibilidad de obtener los alimentos necesarios que les permitirán sobrellevar de mejor forma el período de invierno o pukem.

Por último, el we xipantu es considerado como el período de tiempo que marca el fin y el comienzo de un nuevo ciclo anual, que se establece por medio de la observación de la traslación del sol y que también marca los ciclos estacionales pukem, pewü y walüg. El we xipantu comienza en el período del pukem y se vincula con los cambios en la naturaleza, las actividades sociales y espirituales de celebración del nuevo ciclo anual. Al respecto, un kimche señala: "el we xipantu es el nuevo año de los mapuches, esa fecha tiene mucho significado debido a que ese día vuelve el sol, esa noche vuelve hacia atrás, para así alargar los días" (K1L [216:218]). Es decir, el sol marca el comienzo del ciclo de invierno interpretado como "ese día vuelve el sol". En el contexto de la vida familiar y comunitaria se relaciona con la observación realizada con referentes naturales como los cerros por ejemplo. Por último, para la celebración del we xipantu se efectúan reuniones sociorreligiosas familiares y en comunidad, lo que permite a las familias expresar lazos de unión, creencias y sentimientos respecto del período en el cual se encuentran.

\section{Discusión}

Los resultados permiten revelar la existencia de categorías de contenidos socioculturales que pueden aportar al cuestionamiento de los contenidos del currículum escolar, desde la perspectiva del diálogo de saberes entre el conocimiento sociocultural mapuche y los contenidos disciplinarios (Gasché 2010b; Quilaqueo, 2010; Quintriqueo et al. 2011). En este sentido, los contenidos educativos que utilizan las familias mapuches se sustentan en una lógica de conocimiento que determina el origen y parentesco de las personas en directa relación con los aspectos territoriales, culturales y sociales. A su vez, se constata la vigencia de una concepción de tiempo que se caracteriza por ser cíclica y que se configura por medio de la observación de referentes de la naturaleza y actividades sociales vinculadas con las estaciones del año pukem, pewü y walüg.

El desafío que implica la complementación de estos dos tipos de conocimientos en el aula es que los docentes deben ser algo más que técnicos (Giroux 1998). Es decir, se requiere de personas comprometidas que estén dispuestas a vincular 
los contenidos de enseñanza con el modo de vida de los niños y jóvenes, para así disponer de una nueva alternativa de formación de los estudiantes de contextos multiculturales. Se requiere, entonces, de una educación intercultural con un enfoque crítico, puesto que en el contexto escolar chileno la formación es monocultural y ha propiciado la marginación de los saberes y conocimientos mapuches de la escolarización de las nuevas generaciones (Forno et al. 2009; Quilaqueo et al. 2005). Consecuentemente, estos aspectos debieran ser analizados en conjunto con los distintos actores del medio escolar, para así evitar los conflictos de identidad y los problemas de aprendizaje de los alumnos de origen mapuche, como producto de la negación que experimentan de su patrimonio cultural cuando ingresan al sistema escolar (Quilaqueo et al. 2005; Quintriqueo 2010). En síntesis, se constata que es vital superar la falta de pertinencia de los contenidos utilizados en la formación de los estudiantes provenientes de familias y comunidades mapuches. Por ello, se reconoce que la contextualización curricular debiera incorporar los marcos epistemológicos que sustentan las relaciones sociales, culturales y espirituales de las personas en sus contextos de vida familiar y comunitaria (Quilaqueo et al. 2005; Quintriqueo 2010; Quintriqueo y Cárdenas 2010).

La contextualización se refiere a la incorporación de contenidos educativos propios de la educación familiar mapuche, tanto en las escuelas de las comunidades como en aquellas que se encuentran en los centros urbanos. Es el caso del küpan o ascendencia parental y del tuwün u origen territorial, cuya finalidad educativa es la formación para la acción socioeducativa de los individuos en cuanto al establecimiento de alianzas sociales y familiares, uniones matrimoniales, orientación y conocimiento de las características geográficas del lugar de asentamiento familiar y la espiritualidad que el territorio conlleva. Por otra parte, los conceptos de tiempo del ciclo anual y del we xipantu implican el aprendizaje de las estaciones del año, de los sucesos naturales que en este se desarrollan y del reposo y renacer de la vida en sus distintas expresiones. Además, el aprendizaje de estos conceptos de tiempo incide en la organización de las actividades comunitarias que se desarrollan en cada localidad, por ejemplo, la época de siembra y cosecha. Por lo tanto, con la incorporación de estos conocimientos a las prácticas educativas escolares, se puede contribuir a dar mayor pertinencia a la educación escolar de los estudiantes de comunidades mapuches y también a los no mapuches. Todo esto debería desencadenar la entrega de una formación de mayor pertinencia y calidad, que se hace indispensable para modificar los modelos tradicionales que se han implementado en contextos escolares indígenas, para avanzar hacia una educación intercultural.

El concepto de educación intercultural sugiere la existencia de una pluralidad de culturas en un país, entre las cuales se trata de establecer mejores relaciones, entre el grupo cultural dominante respecto de los grupos indígenas, junto a las distintas comunidades de colonos y emigrados extranjeros en estas últimas décadas. Esta es una concepción que conduce a la pertinencia de introducir una perspectiva intercultural de la educación. Según Ouellet (1991), para instalar la educación intercultural sobre bases sólidas es importante no dejar de lado las nociones de cultura, identidad cultural y relativismo cultural (Camilleri 1988) para comprender los significados que ellas toman en el contexto cultural actual (modernidad, globalización).

En esa perspectiva, según Abdallah-Pretceille (1996), la pedagogía intercultural exige una confrontación permanente de puntos de vista. Al respecto Ouellet (1991) señala que las consideraciones sobre una pedagogía intercultural superan largamente los problemas de educación y de aprendizaje de los alumnos que pertenecen a diversidad multicultural e intercultural, como una toma de consciencia entre la relación educación-cultura y sociedad. Así, desde un paradigma pluralista y relativista, una pedagogía intercultural debería centrar su acción en el reconocimiento y favorecer la coexistencia de las diferencias culturales, aun cuando la finalidad sea mantener la unidad social (Clanet 1988). Basándose en las funciones identificadas por la autora Clanet, la pedagogía intercultural puede ser considerada globalmente como la relación de tres sistemas de significación: (1) Un sistema de significación particular a un grupo determinado, por ejemplo, las significaciones propias de la escuela, lo que constituye un código cultural; (2) Un sistema de significación propio a cada individuo, por ejemplo la de un niño, donde se espera que adquieran el código colectivo transmitido por la escuela, pero donde el sistema de significación particular tributa al medio familiar, a la historia personal, a la vida social; y (3) La transformación de un sistema de significado relativamente cerrado a otro relativamente abierto, es decir, es la perspectiva intercultural de la 
educación para la ciudadanía, de sujetos que están obligados a vivir y transitar desde el mundo global al local y viceversa. En este sentido, a la escuela se le atribuye la responsabilidad en la formación de ciudadanos en el marco de una sociedad democrática, marcada por el multiculturalismo, el pluralismo y la interculturalidad (Dietz y Mateo 2011; Ouellet 2002; Schmelkes 2009), lo que relativiza de alguna manera los conocimientos y obliga a construir un saber multipolarizado, para superar la discriminación y el racismo (Véase, por ejemplo, Essomba 2012; Ouellet 2002; Quintriqueo 2010;). Schmelkes 2009). Por su parte, el currículum intercultural tiene como finalidad la organización y sistematización de contenidos educativos para desarrollar competencias cognitivas, éticas y sociales (Ouellet 2002). En su conjunto, las competencias son capacidades para comprender el mundo en su complejidad sociocultural, basados en principios y valores humanos, seleccionar valores para la actuación ética ligado al derecho, cooperar, reconocer al otro, construir proyectos comunes, desarrollar capacidad para intervenir en la sociedad basado en la justicia social, argumentar y definir situaciones contextuales como base epistémica para la formación de personas en el conocimiento, la autonomía y el reconocimiento (Fornet-Betancourt 2011; Freire 2002; Pizzi 2005; Zuluaga et al. 2003).

En síntesis, basados en nuestras investigaciones y la documentación normativa provenientes del Mineduc (2005) y Unesco (2006), pensamos que las características de un programa de educación intercultural en contexto mapuche requiere situarse en: (1) El diálogo entre sujetos que pertenecen a culturas diferentes; (2) contrarrestar la linealidad de la monoculturalidad; (3) asumir el kimeltuwün en tanto acción educativa mapuche (Quilaqueo et al 2011), como base metodológica para concretar los principios de la educación intercultural, basada en los contenidos y finalidades educativas como una práctica de validación objetiva y subjetiva de lo propio, para superar el desprecio epistemológico del saber educativo mapuche en el contexto escolar; (4) una acción educativa que favorece el descubrimiento de la diversidad cultural, la conciencia del valor positivo de la diversidad cultural y el respeto al patrimonio cultural local; (5) una práctica pedagógica fundada en la conciencia crítica sobre la lucha contra el racismo y la discriminación; (6) un conocimiento acerca del patrimonio cultural local, mediante la enseñanza de la historia, la geografía, la literatura, las lenguas, las artes, los temas científicos y tecnológicos; (7) una comprensión y respeto a todos los pueblos, sus culturas, civilizaciones, valores y formas de vida, comprendidas las culturas indígenas nacionales como de otras naciones; (8) un reconocimiento de la creciente interdependencia mundial de los pueblos y las naciones; (9) una conciencia no solo de los derechos sino también de los deberes mutuos que tienen los individuos, los grupos sociales y nacionales; (10) la colaboración activa y participativa de la familia y la comunidad en la planificación, desarrollo y evaluación de los procesos educativos; (11) que el profesor debe conocer el contexto sociocultural de los alumnos y estar dispuesto a aprender de sus experiencias educativas y socioculturales; y (12) que el profesor debe estar consciente de los propios valores culturales que orientan la interpretación de las situaciones y problemas, así como la capacidad para reflexionar sobre la información y reevaluarla a la luz de diferentes perspectivas culturales y respetando diferentes patrones de pensamientos.

\section{Conclusiones}

El análisis y discusión de los resultados de este estudio permiten afirmar que es necesario implementar una educación intercultural fundamentada en una epistemología que considere tanto los conocimientos educativos familiares, que son la base de la formación de niños y jóvenes mapuches como los conocimientos escolares. De esta forma, se reconoce que la responsabilidad de los actores comprometidos en la formación de los alumnos es clave para crear cambios sustanciales en la educación escolar y es en la construcción de un diálogo de saberes donde se encuentra la alternativa que favorece la generación de una relación intercultural entre el conocimiento mapuche y el escolar. En este sentido, se asume que la escuela basada en un enfoque educativo intercultural debiera superar el nivel discursivo para comenzar a implementar una formación abierta y reflexiva que permita a las nuevas generaciones mapuches y no mapuches formarse para dialogar con el otro en un marco de respeto de las personas, su pertenencia social y cultural.

Finalmente, la necesidad de seguir avanzando en la construcción epistemológica de un cuerpo de conocimientos que sustente la formación de ciudadanos interculturales se plantea como un gran desafío de la escuela intercultural. Para ello, 
se considera fundamental incorporar al marco del estudio nuevas áreas territoriales mapuches que permitan comparar los conceptos ya develados para, así, profundizar en nuevos saberes y conocimientos que están vigentes en la memoria social de los kimches, lo que permitiría a los docentes desarrollar procesos educativos contextualizados a la vida sociocultural mapuche.

Agradecimientos: Agradecemos al Fondo Nacional de Desarrollo Científico y Tecnológico
FONDECYT por el financiamiento de los proyectos de investigación No 1110677 "Tipificación de los métodos educativos mapuches: bases para una educación intercultural” y No 1110489 "Epistemología del conocimiento mapuche y escolar: un análisis desde la pedagogía intercultural, la geografía y sociología educacional". Asimismo, agradecemos a cada kimche participante en el estudio, por las entrevistas sostenidas y su disposición a compartir sus conocimientos. Finalmente, agradecemos a los evaluadores anónimos.

\section{Referencias Citadas}

Abdallah-Pretceille, M. 1996. Vers une Pédagogie Interculturelle. Anthropos, Paris.

Beillerot, J. y N. Mosconi 2006. Traité des Sciences et des Pratiques de l'Éducation. Dunod, Paris.

Bertely, M. 2006. La construcción desde abajo de una nueva educación intercultural bilingüe para México. En La Educación que México Necesita: Visión de Expertos, editado por L. Todd y V. Arredondo, pp. 29-41. Centro de Altos Estudios e Investigación Pedagógica, México.

Bisquerra, R. 2000. Métodos de Investigación Educativa Guía Práctica. Ceac, Barcelona.

- - - 2004. Metodología de la Investigación Educativa. La Muralla, España.

Corbetta, P. 2003. Metodología y Técnicas de Investigación Social. McGrawHill, España.

Camilleri, C. 1988. Pertinence d'une approche scientifique de la culture pour une formation par l'éducation interculturelle. En Pluralisme et École. Jalons pour une Approche Critique de la Formation Interculturelle des Éducateurs, édité par F. Ouellet, pp. 565-594. Institut Québécois de Recherche sur la Culture, Québec.

Clanet, C. 1988. Réflexions sur les fondements possibles d'une pédagogie interculturelle. Pluralisme et École. Actes d'un colloque sur l'éducation interculturelle, 469-493. Université de Sherbrooke, Québec.

Da Silveira, Y., G. Maheux, D. Simard y D. Quilaqueo 2002. Construction de savoirs professionnels et échanges interculturels en contexte de formation des enseignant-es inuit et mapunche. Collectif interculturel. La Revue de l'Institut de Recherche et de Formation Interculturelles de Québec 5:167-185.

Dietz, G. y L. Mateos 2011. Interculturalidad y Educación Intercultural en México: Un Análisis de los Discursos Nacionales e Internacionales en su Impacto en los Modelos Educativos Mexicanos. Secretaría de Educación Pública, México, D.F.

Donoso, A. 2008. Educación y Nación al Sur de la Frontera: Organizaciones Mapuche en el Umbral de Nuestra Contemporaneidad, 1880-1930. Pehuén Editores, Santiago.

Essomba, M. 2012. Interculturalidad en Clave Internacional. Implicaciones para la Educación Indígena. Conferencia dictada en la Facultad de Educación de la Universidad Católica de Temuco. Enero de 2012.

Flores, J. y A. Azócar 2006. Fotografía de Capuchinos y Anglicanos a principios del siglo XX: la escuela como instrumento de cristianización y chilenización. Memoria Americana 14:75-87.

Forno, A., P. Álvarez-Santullano y R. Rivera 2009. Entre el edificio y el currículum de la interculturalidad: una mirada antropológica a la educación actual en territorio mapuche-huilliche. Chungara Revista de Antropología Chilena 41:287-298.

Fornet-Betancourt, R. 2004. Crítica Intercultural de la Filosofía Latinoamericana Actual. Editorial Trotta, Madrid.

- - - 2011. La Filosofía Intercultural y la Dinámica del Reconocimiento. Conferencia Inaugural de la Cátedra Fray Bartolomé de Las Casas, Universidad Católica de Temuco, Temuco.

Freire, P. 1997. Pedagogía de la Autonomía. Siglo XXI editores, México D.F.

- - - 2002. La pedagogía de la Esperanza. Un Encuentro con la Pedagogía del Oprimido. Quinta edición. Siglo XXI, México D.F. - - - 2007. Pedagogía del Oprimido. Siglo XXI, México D.F.

Gasché, J. 2002. Criterios e instrumentos de una pedagogía intercultural para proyectos de desarrollo en el medio bosquesino Amazónico. Relaciones 23(91):193-234. Colegio de Michoacán, Zamora.

- - - 2010a. De hablar de la educación intercultural a hacerla. Mundo Amazónico 1:111-134. Universidad Nacional de Colombia, Bogotá.

- - - 2010b. ¿Qué son "saberes” o "conocimientos” indígenas, y qué hay que entender por "diálogo"? En Memorias Primer Encuentro Amazónico de Experiencias de Diálogo de Saberes. Leticia 10 al 12 de noviembre de 2008, editado por C. Pérez y J. Echeverri, pp. 16-30. Universidad Nacional de Colombia, Sede Amazonía.

Giroux, H. 1998. Sociedad, Cultura y Educación. Miño y Dávila Editores, Madrid.

- - - 2004. Teoría y Resistencia en Educación. Siglo XXI, México D.F.

Maldonado, N. 2007. Sobre la colonialidad del ser: contribuciones al desarrollo de un concepto. En El Giro Decolonial. Reflexiones 
para una Diversidad Epistémica más allá del Capitalismo Global, editado por S. Castro-Gómez y R. Grosfoguel, pp. 127-167. Iesco-Pensar-Siglo del Hombre Editores, Bogotá.

Ministerio de Educación de Chile (MINEDUC) 2005. Orientaciones para la contextualización de planes y programas para la Educación Intercultural Bilingüe. MINEDUC, Santiago.

McMillan, J. y S. Schumacher 2005. Investigación Educativa. Pearson Educación, Madrid.

Moscovici, S. 1983. Influencia manifiesta e influencia oculta en la comunicación. Revista Mexicana de Sociología 2:687-701.

Ouellet, F. 1991. L'éducation Interculturelle. Essai sur le Contenu de la Formation des Maîtres. Editions L'Harmattan, Paris.

- - - 2002. L'éducation interculturelle et l'éducation à la citoyenneté. Quelques pistes pour s'orienter dans la diversité des conceptions. Ville École Intégration Enjeux 129:146-167.

Pérez, E. y N. Alfonzo 2008. Diálogo de saberes y proyectos de investigación en la escuela. Educere 42:455-460.

Pizzi, J. 2005. El Mundo de la Vida. Husserl y Habermas. Ediciones Universidad Católica Silva Henríquez, Santiago.

Plata, J. 2010. Investigación social y diálogo de saberes. En Memorias Primer Encuentro Amazónico de Experiencias de Diálogo de Saberes. Leticia 10 al 12 de noviembre de 2008, editado por C. Pérez y J. Echeverri, pp. 31-40. Universidad Nacional de Colombia, Sede Amazonía.

Quilaqueo, D. 2006. "Valores educativos mapuches para la formación de persona desde el discurso de kimches". Estudios Pedagógicos 32(2):73-86.

- - - 2007. Saberes y conocimientos indígenas en la formación de profesores de educación intercultural. Educar em Revista 29:223-239.

- - - 2009. Interculturalidad en contexto mapuche. Revista Cultura-Hombre-Sociedad 17(1):11-19.

- - - 2010. Racionalidad de los saberes educativos mapuches apoyada en la memoria social de los kimches. En Interculturalidad en Contexto Mapuche, editado por D. Quilaqueo, C. Fernández y S. Quintriqueo, pp. 55-82. Editorial EDUCO, Neuquén.

- - - 2012. Saberes educativos mapuche: racionalidad apoyada en la memoria social de los kimches. Atenea 505:70-102.

Quilaqueo, D. y M. Merino 2003. Estereotipos y prejuicio étnico hacia los mapuches en textos complementarios a la asignatura de Historia. Campo Abierto 23:119-135. Badajoz.

Quilaqueo, D. y S. Quintriqueo 2008. Formación docente en educación intercultural para contexto mapuche en Chile. Cuadernos Interculturales 6(10):91-110.

- - - 2010. Saberes educativos mapuches: un análisis desde la perspectiva de los kimches. Polis Revista de la Universidad Bolivariana 9(26):337-360.

Quilaqueo, D., S. Quintriqueo y P. Cárdenas 2005. Educación, Currículum e Interculturalidad. Elementos sobre Formación de Profesores en Contexto Mapuche. Frasis editores, Santiago.
Quilaqueo, D., S. Quintriqueo y D. San Martín 2011. Contenido de aprendizajes educativos mapuches para el marco conceptual de un currículum escolar intercultural. Estudios Pedagógicos 37(2):233-248.

Quintriqueo, S. 2010. Implicancias de un Modelo Curricular Monocultural en Contexto Mapuche. Editorial LOM, Temuco.

Quintriqueo, S. y P. Cárdenas 2010. Educación intercultural en contextos mapuches: hacia una articulación entre el conocimiento mapuche y conocimiento disciplinario en ciencia. En Interculturalidad en Contexto Mapuche, editado por D. Quilaqueo, C. Fernández y S. Quintriqueo, pp. 89-126. Editorial EDUCO, Neuquén.

Quintriqueo, S. y M. McGinity 2009. Implicancias de un modelo curricular monocultural en la construcción de la identidad sociocultural de alumnos/as mapuches de la IX región de La Araucanía, Chile. Estudios Pedagógicos 35(2):173-188.

Quintriqueo, S. y H. Torres 2013. Construcción de conocimiento mapuche y su relación con el conocimiento escolar. Estudios Pedagógicos 39(1):199-216.

Quintriqueo, S., H. Torres, M. Gutiérrez y D. Sáez 2011. Articulación entre el conocimiento cultural mapuche y el conocimiento escolar en ciencia. Educación y Educadores 14:475-492.

Quivy, R. y L. Campenhoudt 1998. Manual de Investigación en Ciencias Sociales. Limusa-Noriega Editores, México D.F.

Ruiz Olabuénaga, J. 1996. Metodología de la Investigación Cualitativa. Universidad de Deusto, Bilbao.

Schmelkes, S. 2009. Interculturalidad, democracia y formación valoral en México. Revista Electrónica de Investigación Educativa 11(2):10-08.

Stake, R. 2005. Investigación con Estudio de Casos. Morata, Madrid.

Tubino, F. 2011. El nivel epistémico de los conflictos interculturales. Construyendo Nuestra Interculturalidad 6/7:1-14.

UNESCO Organización de las Naciones Unidas para la Educación, la Ciencia y la Cultura 2006. Directrices de la UNESCO sobre la educación intercultural. Sección de Educación para la Paz y los Derechos Humanos, División de Promoción de la Educación de Calidad Sector de Educación. UNESCO, Paris.

Walsh, C. 2005. Interculturalidad, conocimientos y decolonialidad. Signo y Pensamiento 24(46):39-50.

- - - 2008. Interculturalidad, plurinacionalidad y decolonialidad: las insurgencias político-epistémicas de refundar el Estado. Tabula Rasa 9:131-152. Bogotá.

Wallen, N. y J. Fraenkel 2001. Educational Research: A Guide to Process. Lawrence Erlbaum Associates, Inc., New Jersey.

Zuluaga, O., A. Echeverri, A. Martínez, H. Quiceno, J. Sáenz y A. Álvarez 2003. Pedagogía y Epistemología. Magisterio Editorial, Bogotá. 


\section{Notas}

* Este artículo es producto de la colaboración entre el Núcleo Milenio Centro de Investigación en Educación en Contexto Indígena e Intercultural (CIECII), Universidad Católica de Temuco; el Núcleo de Estudios Interétnicos e Interculturales (NEII), Universidad Católica de Temuco; y el Centre Interuniversitaire d'Études et de Recherches Autochtones (CIÉRA) de l'Université Laval, Québec, Canada.

1 El diálogo de saberes de poblaciones indígenas de América Latina ha sido cuestionado por los dirigentes indígenas al verse minorizados por los científicos o utilizados para construir categorías a partir de disciplinas que los han excluido por tratarse de conocimientos exclusivamente orales (Gasché 2010b; Plata 2010).

2 Área territorial wenteche: Maquehue, comuna de Padre Las Casas; Ragiñtulewfu, comuna de Nueva Imperial; Chumil y Llamuco, comuna de Vilcún. Área territorial nagche: Lipun, comuna de Galvarino; Quilquen, comuna de Los Sauces; Repocura, comuna de Cholchol. Área pewenche: Cayaqui, comuna de Alto Biobío. 
